\title{
GSM and IoT based Smart Traffic System
}

\section{with Geofencing}

\author{
Ghouri $^{1}$, Malahim ${ }^{1}$, Fatima $^{1}$ Tayyab $^{1}$, Shakir ${ }^{1}$,
}

\begin{abstract}
This project is aimed at resolving severe traffic congestion in most cities across the world by using latest technologies. The world is heading towards IoT and shifting daily routine manual processes to automatic systems. Current traffic control system is based on fixed timer which becomes one of the main reasons of transport blockage. In order to overcome this problem, a framework has been designed to introduce the concept of smart traffic system which includes Internet of Things. The road side sensors attached to the Arduino Mega send information to the cloud using NodeMCU where decision is taken based on density, hence involving cloud computation to turn a particular signal green. Moreover, we have also dealt with emergency vehicles which bears the facility of turning signal green using either RFID system or GSM based mobile. Sound sensors are placed to confirm that the signal return to normal condition once the emergency vehicle has crossed the signal. Lastly, a geofencing based marketing app called "Brando" has been designed using android studio to provide location-based services from the nearest stations like shopping malls to the people on road on their respective mobile phones.
\end{abstract}

Keywords- IoT, Density, Traffic Signal, Road Side Sensors, Emergency-Vehicles, Marketing.

\section{INTRODUCTION}

Traffic light at multi-way junctions in many metropolitan cities are functioned to allow entry with fixed timing, hence, providing constant time period every time and not considering current situation. For example, in the rush hours of morning, usually heavy traffic flow is observed on junctions providing path to the commercial area whereas the traffic signal installed in the path to residential areas are found to be less loaded. Traffic light in such situations allocate same time duration for traffic to move in both direction which becomes ineffective when it comes to the vehicles going into commercial area.

We proposed this project in order to resolve the traffic issue found at signals. The whole system will receive signals from the Road Side Units (RSU) and send it to the cloud for the computation of time parameters for Traffic Lights. Different sensors have been used to count the number of vehicles. Moreover, we have also designed the project to deal with emergency vehicles. RFID system placed at some distance away from the signal will allow emergency vehicle's driver to turn the signal green until he passed the road. The system will return to normal condition once the vehicle siren is detected by the sound sensor. Here we also made assure that system works on priority basis. Moreover, even if the driver misses the RFID, he can still use mobile phone to activate the signal to GSM module attached to the signal to open the particular pathway.

${ }^{1}$ Department of System Engineering, Hamdard University, Karachi, Pakistan.
Since all GSM mobile phones are GPS enabled so by using the following technology, we can detect the presence of a customer through the installed app named "Brando" and using push notification we can send product deals available in the nearby malls and shopping centers as soon as he/she enters the geofenced area. Push notifications are messages displayed on mobile devices. App providers can send these notifications to gadgets at any time. Users don't have to enter the application or use their mobile to get it. To test the above concept, we have used the GPS co-ordinates of several departments like Bait Ul Hikmah, Sports Center, HCMD and Fest that are present inside Hamdard University (Main Campus, Karachi).

\section{CHALlEnges FOR PROPOSED SYSTEM}

\section{A. Challenges for Density Based Traffic System}

Generally there are two phases through which the whole system is going to work. First is to detect general vehicles through RSUs and other is to detect Emergency Vehicles.

\section{B. Challenges for Location Based Advertisement}

Firstly, an individual should be able to use the app easily. The user should have given permission for the use of his location and as the user enter a geo-fence, push notification for the product detail must be reached.

\section{TRAFFIC CONGESTION}

In the transportation community, congestion relates to an excess of vehicles on a portion of roadway at a particular time, resulting in speeds that are slower than free-flow speeds (Cambridge Systematics, Inc., 2005). The physiological phenomenon found among human relates that completing the journey in a longer time than anticipated is taken to imply that the road was congested.

\section{A. Causes}

The general reasons of traffic jam consist of: too many cars traveling on an avenue, variant inside the capability of avenue infrastructure, driving sample of driver, employment patterns, and economic standard of a society. Below are the three clusters that can be concluded as one of the reasons for bottleneck.

- Macro-level dynamics: Causes of traffic that are directly related to the status of the road.

- Micro-level dynamics: These relate to causes that are associated with demand of road facilities.

- Indirect dynamics: Random variables that contribute to the commencement of congestion such as weather and events. 


\section{B. Costs}

Costs of congestion includes reduced speeds and increased travel times. Figure 3.0 below shows how traffic jam can be detrimental to a society. The costs that result due to congestion can be subdivided into two categories.

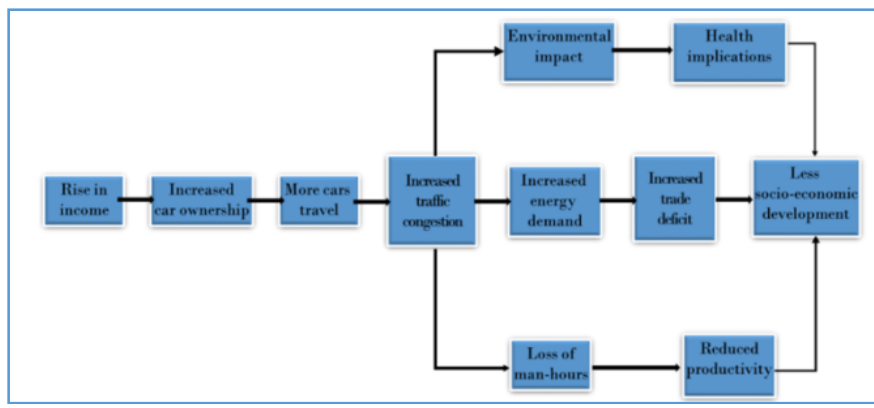

Figure 3.0: Impacts of congestion

- Direct Cost: The cost of auto-mobile maintenance that take place due to longer stay in traffic jam.

- Indirect Cost: The cost that occurs as loss of life due to accidents and increased commodity prices.

\section{Congestion Management Procedure.}

Two strategies that are designed mainly to overcome with obstruction are present below:

- Fixed: The fixed traffic signal light works with preset timing, the duration of the signal displayed on the light is preset.

- Real time: This technology allows stop signals to take decisions derived from the current traffic situation.

\section{Wireless Sensor Networks (WSNs)}

The information gathered from the environment through network of sensors to communicate wirelessly over the network through a gateway is known as WSNs. The nodes for WSNs are comparably cheap when dealing with the amount of data they are able to collect. Figure below displays the sensors in a network.

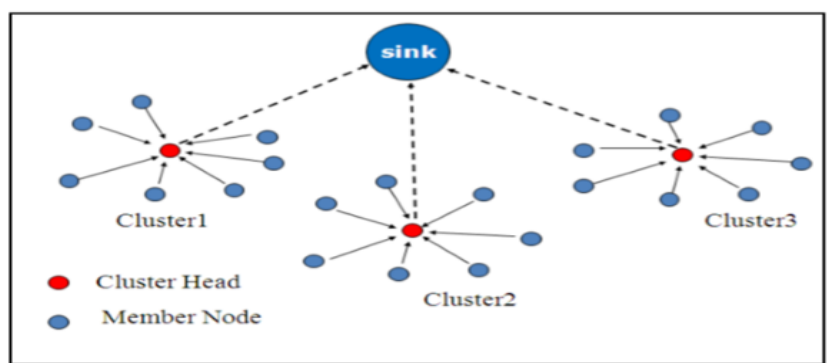

Figure 3.1: Sensors in a network

\section{PHASES INVOLVED IN PROJECT}

The section deals with the population and sampling techniques along with the instruments that were used to collect and analyze the information received. It also includes methods used to maintain validity and reliability of the instrument. The research was completed in four phases which are described below.
Information in phase 1 was obtained using a qualitative approach, while phase 2 can be considered as quantitative. However, Phases 3 was focused on designing Location Based Application. Phase 4 was to test the complete hardware and software system using the verified methods.

\section{A. Phase 1}

This phase involved finding out views and perceptions of road users about congestion in Karachi. The data for this phase was collected through live questionnaire.

B. Phase 2

The goal of phase 2 was to look for the methods available worldwide to count vehicles and sending the collected data to the cloud.

C. Phase 3

This phase involved the designing of the geofencing app and integrating the google map API with GeoFire. At the end of this phase, the application was debugged to bring any changes, if necessary.

Algorithm: The formulae presented below not only helps in the creation of our mobile geofencing application but also in providing the ratio of people receiving the particular ads in the given radius:

Where,

$$
\mathrm{Z}=[\mathrm{Nr} \cdot \mathrm{p} / \mathrm{y} * \mathbf{y}] / \mathrm{p}
$$

$\mathrm{Z}=$ No of Forecasted Clients

$\mathrm{r}=$ Radius of Geo-fence $(\mathrm{km})$

$\mathrm{p}=$ Total No of People

$\mathrm{N}=$ No of Ads

$\mathrm{y}=$ Time consumed 


\section{Phase 4}

Phase 4 involved the testing of the complete system which includes both hardware and software.

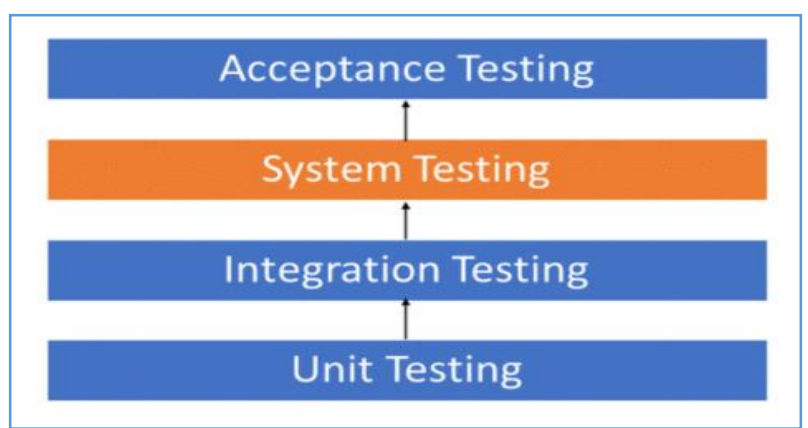

Figure 4.1: Software Testing Hierarchy

\section{Project Working Methodology}

Arduino Mega receives input from HMC5883L, IR Sensors and PNP Inductive Proximity Sensors. The data from these sensors includes the information about the number of vehicles present on a particular lane of the signal. This data is sent from the Mega to NodeMCU which further connects the system with internet technology, hence allowing the data to be stored in Firebase database and can be observed on local server. The data is analyzed and the time for the signal to turn green is calculated using the devised algorithm. The signal is then sent from NodeMCU to Mega which further sent it to the TM1637. The values displayed on the timer is due to the density.

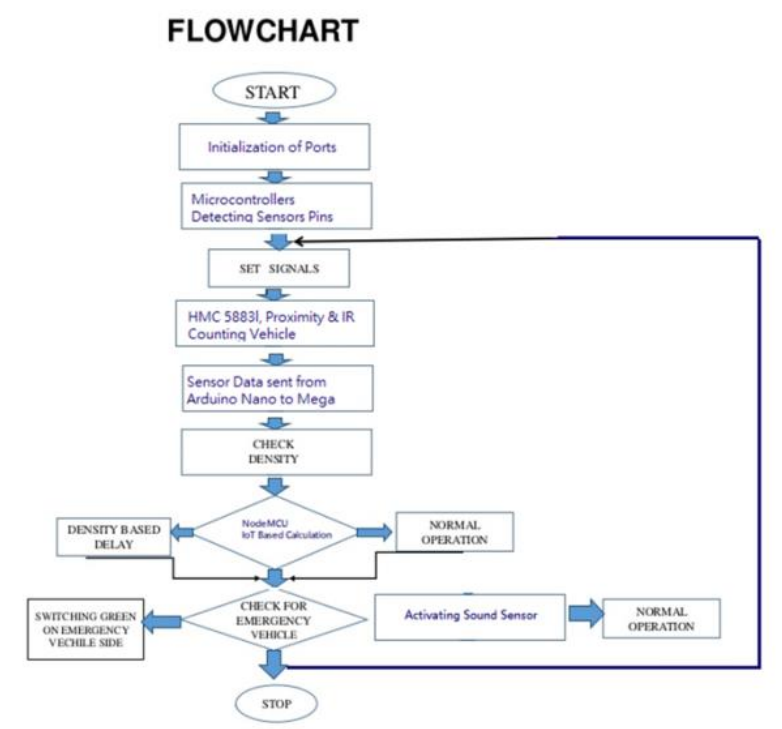

The interruption to the system arrives when it detects an emergency vehicle either from the RFID or GSM Module. RFIDs Tags are provided to the drivers of emergency vehicles so that they can place it on RFID Modules placed around $500 \mathrm{~m}$ away from the signal. These modules work on the priority basis and turn the signal green immediately depending, which module on a particular road is triggered first while turning the remaining signal red until the sound sensor placed on the other side of the road detects the vehicle siren. In case of heavy traffic jam or missed RFID Module, the system can also be triggered by making call to the GSM modules connected with the signal. The timing of TM1637 varies in such scenarios with a longer time duration, to confirm the crossing of such vehicles more efficiently.

A. Block Diagram of IoT Based Smart Traffic System

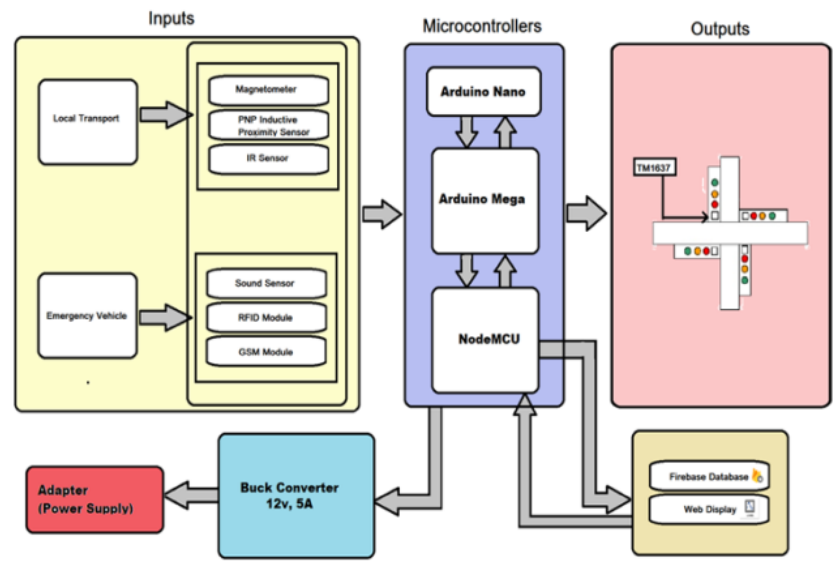

B. Understanding Geofencing Geographically

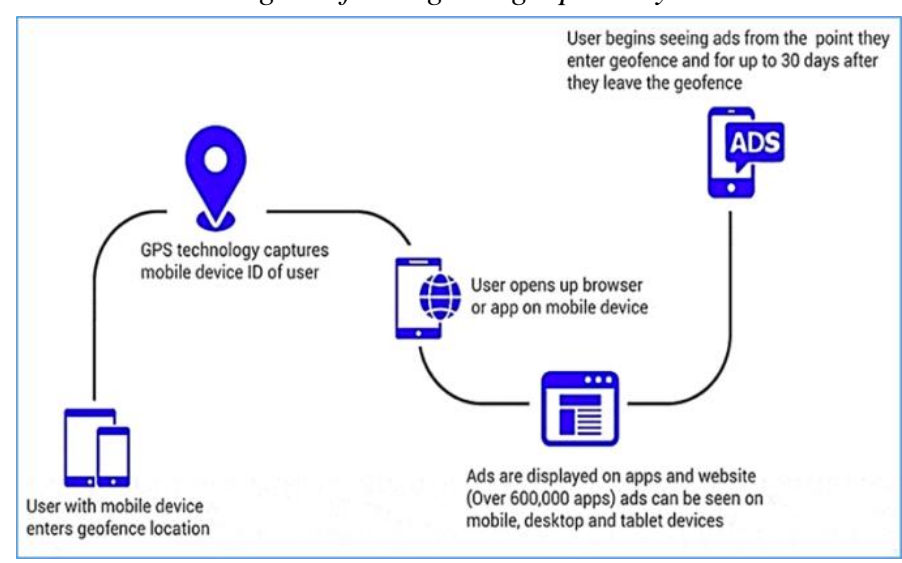

\section{Techniques \& Modules used to Identify Emergency Cases}

The technique used to detect such vehicles is by providing them with RFID tags and a special mobile number. RFID modules installed $500 \mathrm{~m}$ away from the signal on the fastest lane on the driver's side allows that vehicle to turn signal on for a specific time on placing RFID key. In case of traffic jam or if the driver missed the RFID, the solution is to call on the GSM module attached with that signal. This would generate a signal that would be sent to the Arduino which will turn the signal green. Both of the techniques are efficient and reliable.

\section{Geofencing App for Hamdard University}

Geofencing App is designed and tested in Hamdard University to facilitate the students and visitors to get particular information about the department, once the crossed the fenced area. This feature of the app is available to both Android and IOS Users.

a) Creating user alert on Entrance, Dwelling and leaving Fenced area.

The Push Notification services are enabled to work even if the app is working on background on both Android and IOS. This will led the user to get latest information from the company on entering, dwelling or leaving the fenced area. This project is implemented in Hamdard University by providing the fixed co-ordinates of Bait-ul-Hikmah, Sports Complex, HIET and Dental Department to the designed 
geofenced component of Brando Software. The application makes an alert sound on getting any of the provided condition true. The system of notification is further enhanced in react native by integrating it with One-Signal so that a customized notification can be sent to any particular user at any time. Moreover, this function will work on all the devices which have SDK version above 26

\section{GEOFENCING APPLICATION}

\section{A. Brando}

This user-friendly application is designed to bring awareness in the local public by attracting them with exclusive offers provided by different companies around them. This app has got numerous features which are listed below.

\section{a). Sign In/ Sign Up}

This feature allows a user to have personal account so that his/her private data cannot be accessed by another user.
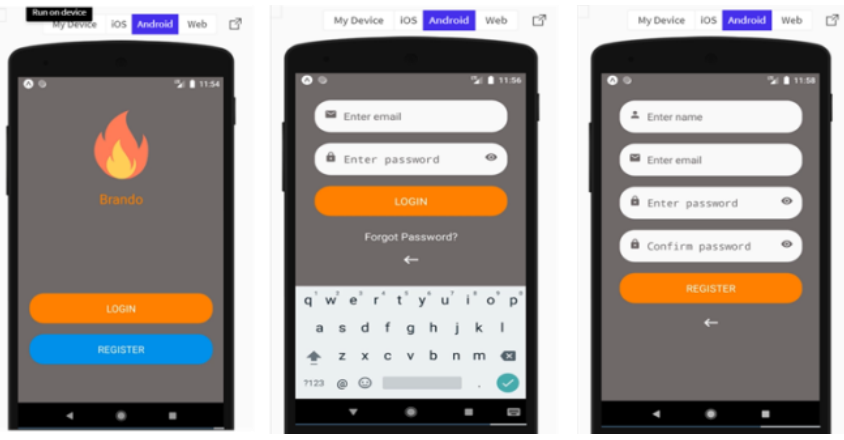

\section{b) Home Screen}

The home screen includes different sections where user is guided to access other features of the app using the bottom tab navigation. These sections are:

\section{Feed Screen}

This screen provides the current news of the city to the user so that he remains aware of any emergency situation while travelling. The news API is fetched in the program.

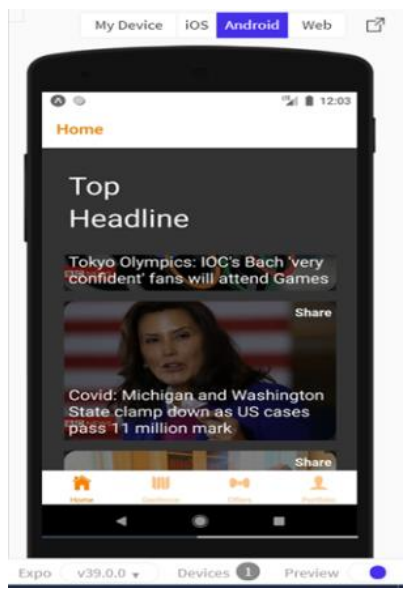

2. Geofencing Screen
This screen shows the user his current location and the areas which are geofenced. It helps the user to get notified about the product once they are in an entering, dwelling or leaving state of a geofenced.
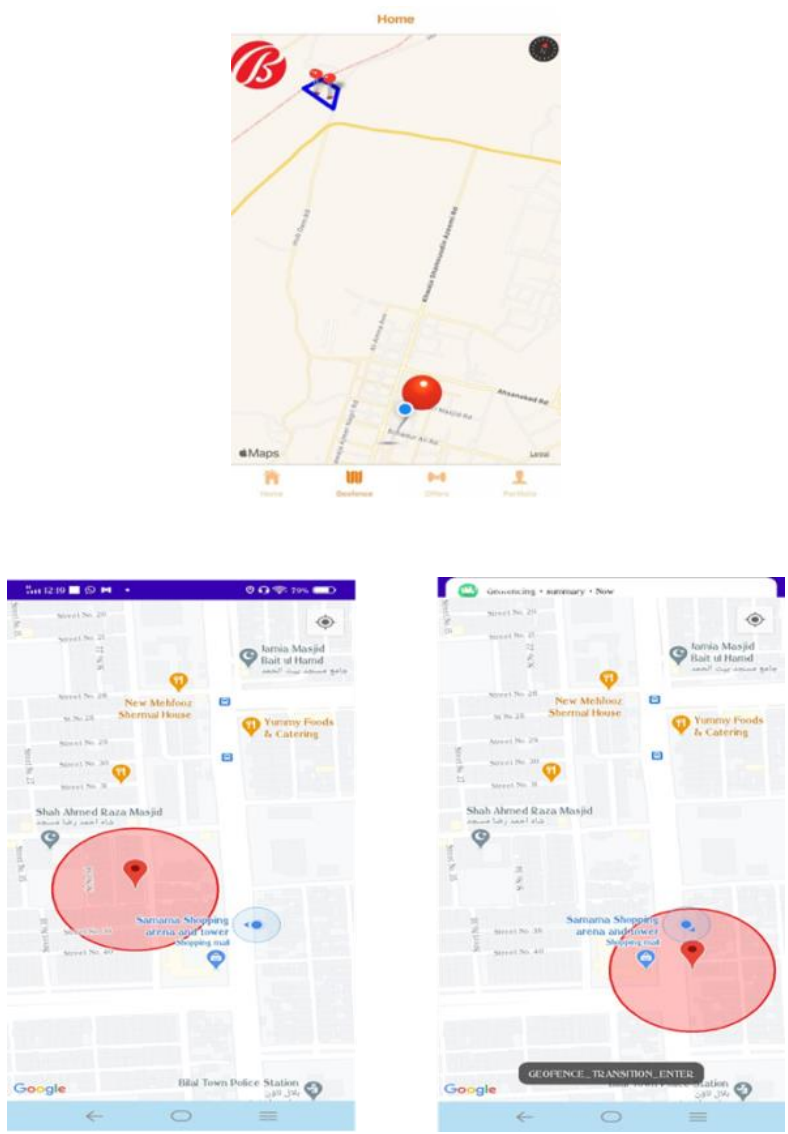

3. Offers

This screen includes a swappable list where the user get awareness about the latest advertisements and offers provided by different brands.

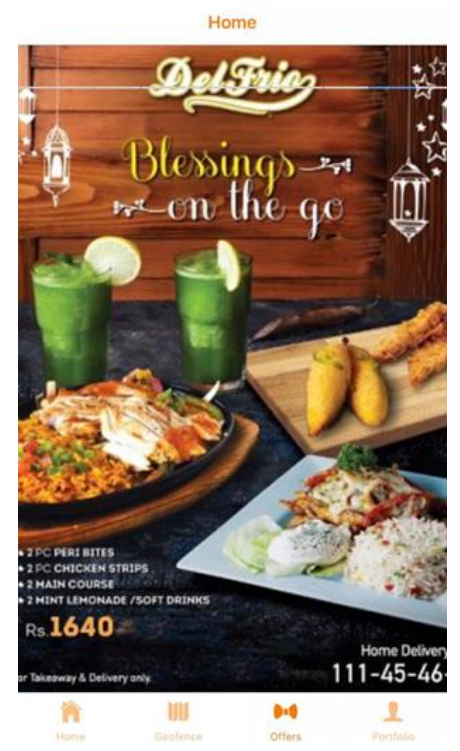

4. Developer Portfolio and Contact Details 
This page provides the option to the user for logging out of the application. Moreover, in case of any suggestion or problem, one can contact us through the provided number or email, for further assistance.

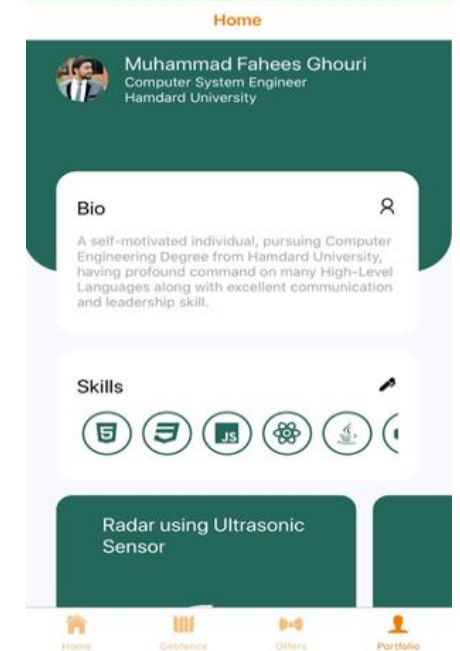

\section{Notifications}

It is one of the main functions when it comes to notifying the user. Push Notification are necessary part of a location-based application. We have used OneSignal API for this. OneSignal is the market leader in customer engagement, powering mobile + web push, email \& inapp messages. Integrating this API with firebase allows a company to promote their deals by sending customized notification on a particular ID.

\section{B. Flowchart and ER Diagram of Brando}

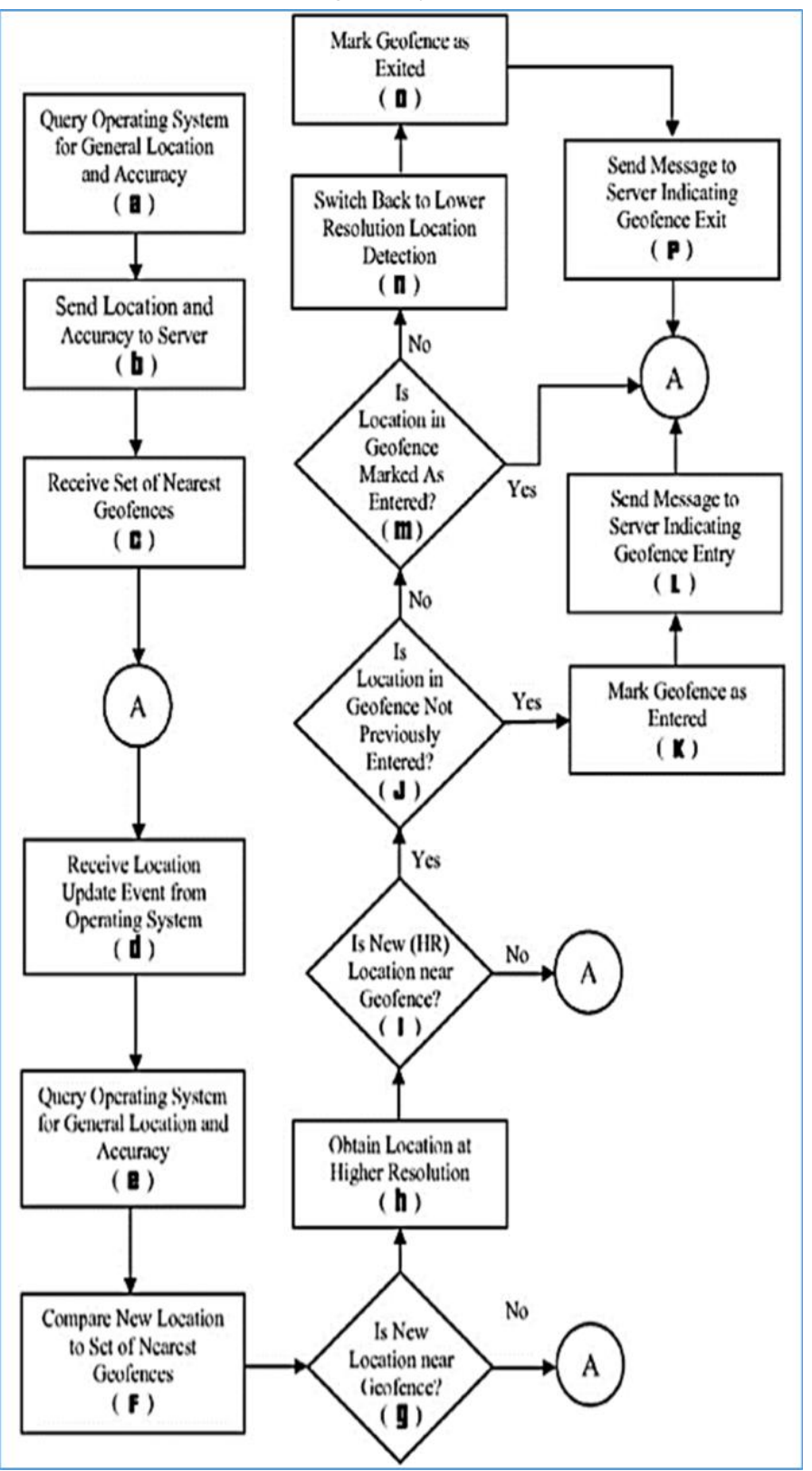




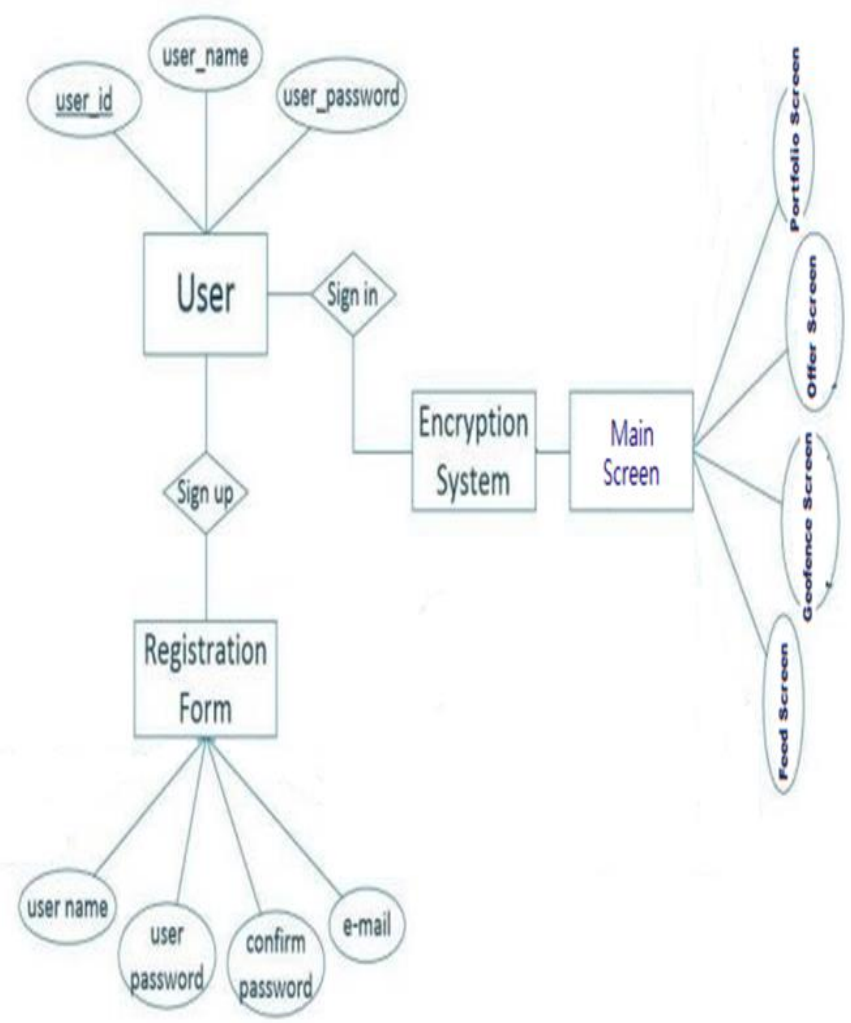

\section{ADVANTAGES OF THE SYSTEM}

1. Easy and efficient surpassing of heavy traffic at signals.

2. Reduces situations like bottleneck on streets.

3. Instantaneous auto-mobile monitoring

4. Awareness of the new product available in the market.

5. Significant increase observed in the economy of a country

6. Immediate traffic clearance on emergency situation using RFID and GSM Module.

\section{OVERVIEW OF SYSTEM}

In this research paper an IoT-based traffic control system is displayed. The major goal of the designed system is to reduce the impacts of congestion including both environmental and societal.

The system is able to provide information related to traffic to drivers on multiple platforms. Moreover, the information regarding advertisement can be 'pushed' to motorists through push notification, hence a helpful parameter in increasing awareness. In this work, road side units are installed to collect traffic parameters from the road. The parameters of interest includes the counting of vehicles passing a particular signal and the time displayed on the signal to remain green in order to avoid congestion. The other parameter that was of interest in this paper was to provide awareness regarding new products and offers available from a particular company to the public once they entered the geofenced area. The devised algorithms helps in the counting of vehicles, adjusting the time of signal to remain green. The data obtained from the algorithm triggers the signals attached to the microcontroller resulting in the delay of time period of signal along with providing the solution to the emergency vehicles.

This project has answered the first research question by confirming that amount of population is directly proportion to the flow of traffic.

The second objective of the research has been responded by providing the cheapest and reliable techniques to count the number of vehicles while considering the economic situation of Pakistan.

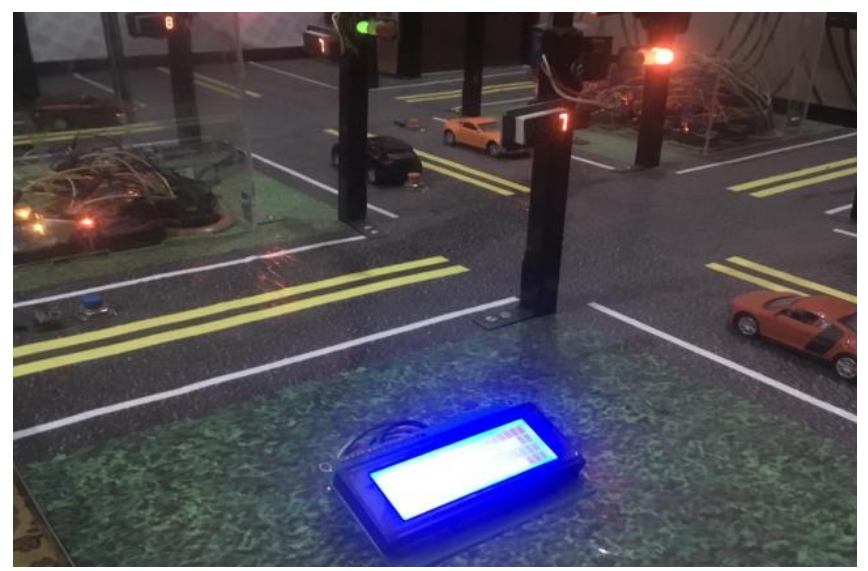

The third research question of providing LBA to public and its working phase was responded by designing of geofencing app which uses the google map API to track the current location of a particular user and comparing it with the fenced area.

The last phase was answered by testing the complete system under the authorized hardware and software analyzing techniques.

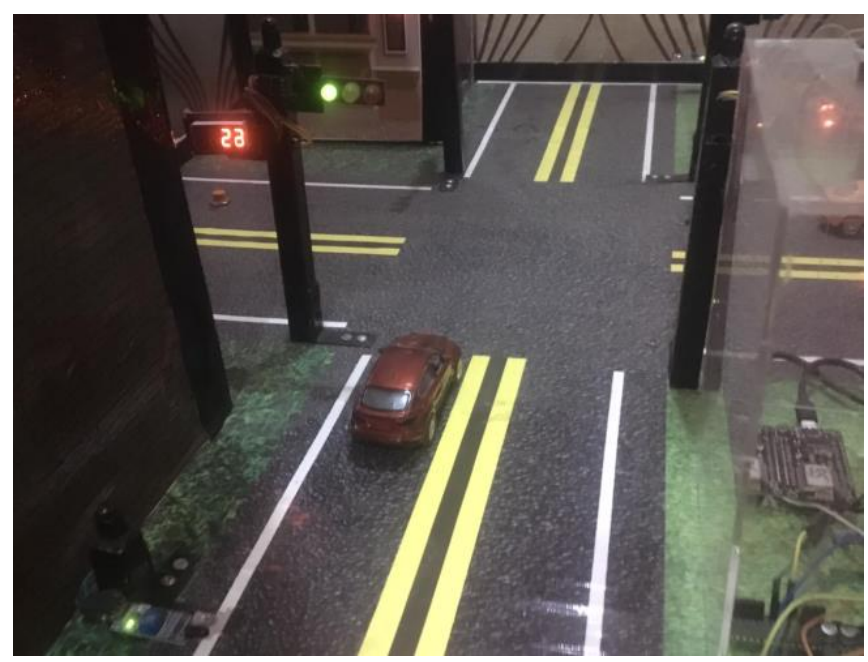




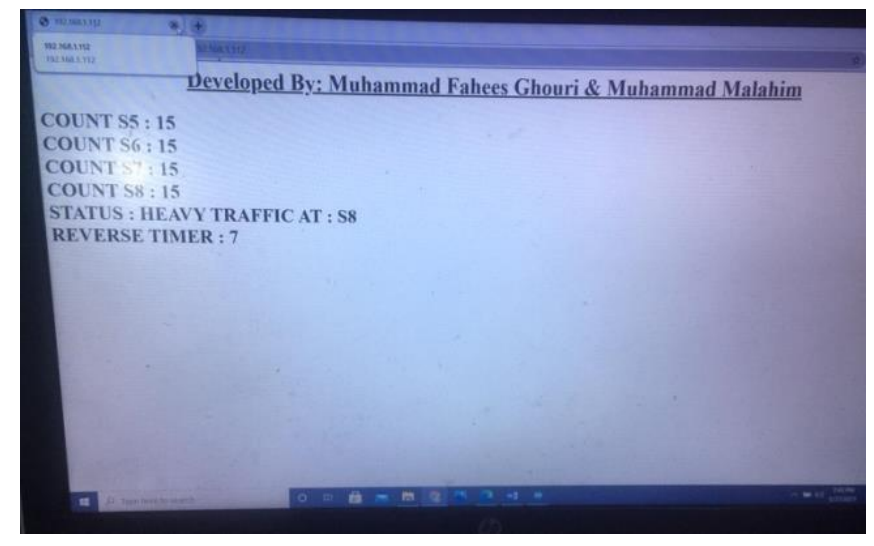

Signal Getting Back To Normal State.

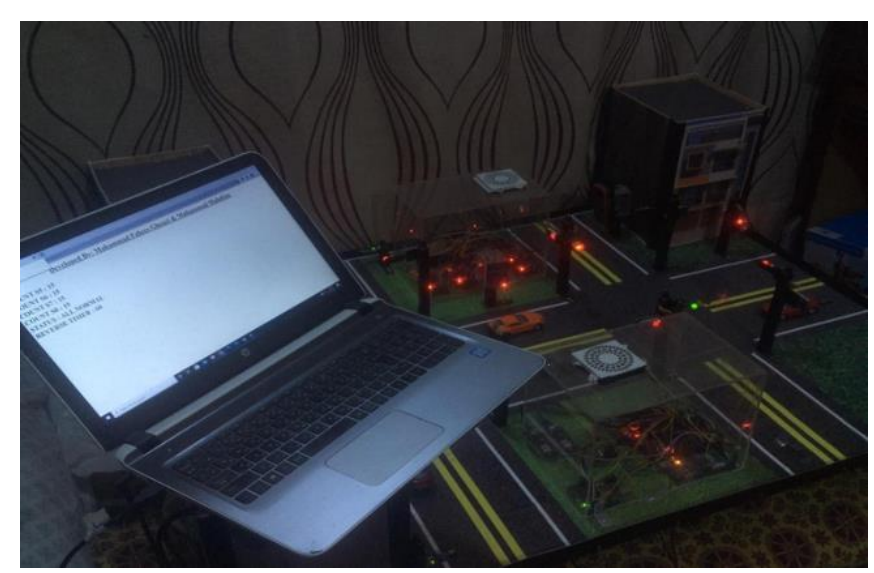

\section{REFERENCES}

[1] Wei-Hsun Lee, and Chi-Yi Chiu, 2021. Design and Implementation of a Smart Traffic

[2] Signal Control System for Smart City Applications.

[3] A Shokhayba Rekh., 2021. Smart traffic management system with real time analysis.

[4] Khalid Chahine \& Bilal Ghazal. 2020. Smart traffic light control system.

[5] MD Khurram Munir., 2020. A Review of IoT Application in a Smart Traffic Management System.

[6] AbuBakr M Miyim, 2019. Smart Traffic Management System.

[7] K.Suresh \& Patan Rizwan, IEEE 2019, Real-time smart traffic management system for smart cities by using Internet of Things and big data

[8] Mamatha Rath, 2018 IOP Conf. Ser.: Mater. Sci. Eng. 377 012201, Smart Traffic

[9] Management System for Traffic Control using Automated Mechanical and Electronic Devices.

[10] Guisippe Guido,2017 Journal of Advance Transportation, Intelligent Traffic Control and Management in Urban Area.

[11] Litman, T., 2016. Smart Congestion Relief Comprehensive Evaluation Of Traffic Congestion Costs and Congestion Reduction Strategies.

[12] Adunya, O., 2015. An Intelligent Traffic Light Control System Based on Fuzzy Logic Algorithm. International Academic Journal of Information Systems and Technology.

[13] Babiker, S. \& Bashi, A., 2015. Security of Wireless Sensor Networks for Monitoring System. American Journal of Computer Science and Engineering.

[14] Litman, T., 2009. Definitions, Costing Methods, and Estimates Techniques, Estimates and Implications. In: Costs - Overview and Definitions. Anon: Victoria Transport Policy Institute. 